ORIGINAL ARTICLE

\title{
Monitoring trends in obesity in South Wales using routine data
}

\author{
S E Jones, M James-Ellison, S Young, M B Gravenor, R Williams
}

See end of article for authors' affiliations

a.t.r.

Correspondence to:

Prof. R Williams, Swansea

Clinical School, University

of Wales Swansea,

Swansea SA2 8PP, UK;

D.R.R.Williams@

swansea.ac.uk

Accepted

12 November 2004
Aim: To use existing data on height and weight of 5 year old children, routinely recorded annually as part of the school entry medical, to monitor trends in obesity over the last 16 years in three South Wales localities.

Methods: Body mass index (BMI) and percentage of children over the cut off points for being overweight or obese proposed by the International Obesity Task Force were determined in 46073 children who had height, weight, and sex recorded each school year (between 1986/87 and 2001/02) on the National Child Health Computer System held at the Swansea NHS Trust.

Results: With the exception of one year, the coverage for BMI measurements ranged between $87 \%$ and $99 \%$. The accuracy of measurement and data entry was identified as suspect in some cases, and although some data entry errors could be corrected, 14\% of BMI measurements were inadmissible. Logistic regression analysis of BMI trends in the remainder showed that the percentage of 5 year olds classified as overweight or obese had increased significantly over the time period and that the rate of change in girls was significantly greater than that in boys.

Conclusion: Overweight and obese children have increased in frequency in this population. The Child Health Computer System is potentially a valuable source of information on the health status of populations and should be capable of monitoring trends in obesity. However, accuracy of measurement and data entry need to be improved, and the system, to be useful on a national basis, needs to be amalgamated at a higher level than individual NHS Trusts.
O besity in childhood is now recognised as a major public health problem associated with significant comorbidities in childhood and increased risk of adult diseases such as cardiovascular disease and type 2 diabetes. ${ }^{1}$ Accurate and comprehensive data are required if we are to monitor trends in childhood obesity and assess the impact of preventive strategies.

One potential source of such population data is the Child Health Computer System. However, a recent study from South Wales $^{2}$ showed poor coverage of height and weight measurements and suggested that further training of health professionals was necessary to make full use of this system for developmental screening. The commentary ${ }^{3}$ which accompanied this article highlighted the need to improve the quality of this database and suggested that public health departments in Primary Care Trusts and Strategic Health Authorities (and, presumably, similar bodies elsewhere in the United Kingdom) should be monitoring data on childhood obesity. The importance of this for the existing Coronary Heart Disease and Diabetes National Service Frameworks (NSFs) and for the forthcoming Children's NSF was underlined.

This study describes an analysis of the local (Swansea, Neath, and Port Talbot) Child Health Computer System records to investigate the practical issues involved in using such data and to describe secular trends in childhood obesity in these localities. The findings are intended to serve as a baseline enabling comparison with trends elsewhere and with future trends within this locality. Approval for the study was given by the local research ethics committee.

\section{METHODS}

The National Child Health Computer System had been introduced locally in 1986. At this time all children in the reception year in primary school had been offered a school entry medical. This involved height and weight measurement by the clinic nurse with the results entered onto the system by administrative staff. In 1990 this routine medical was abandoned in favour of a more selective approach following recommendations such as those of the Hall reports. ${ }^{4}$ Height and weight measurements continued to be recorded following an update on training for clinic nurses. At this time the Leicester height measure was introduced as standard to replace all other equipment in circulation.

Using the Seagate Crystal Reporting Software, results for body mass index (BMI: weight $(\mathrm{kg}) /$ height $\left.(\mathrm{m})^{2}\right)$ in one year (2001/02) were scrutinised for outliers. It was apparent that weight data in particular had sometimes been recorded incorrectly, giving rise to spurious BMI results. These recording errors were usually the result of incorrect placing of decimal points. A correction formula (multiplication of the weight entry by 10) was devised to overcome these. Other unaccountable errors were noted. In order to eliminate the effect of remaining outliers, some of which were also due to incorrect data entry, analysis, for this and all other years from 1986/87, was restricted to a BMI range of 10 to 27 units based on the Child Growth Foundation 1997 BMI reference charts.

During the analysis it was noted that some children were measured on more than one occasion during their reception year. This occurred while being recalled for visual acuity checks. These repeated height measurements were scrutinised for cases in which the second height measurement was less than the first as an indication of the frequency of at least one obvious measurement error.

The Seagate Crystal Reporting Software was then used to calculate (for each year from 1986/87 to 2001/02 and for boys and girls separately) coverage (the proportion of children listed by the system who also had a record of sex, height, and weight), mean BMI, and the proportions lying within the "acceptable" and above the "overweight" and "obese" cut off points. These were as advocated, for 5 year old children, by the International Obesity Task Force (IOTF): for boys, 17.4 and 19.3; and for girls, 17.1 and 19.2. 


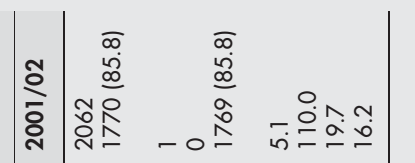

흥

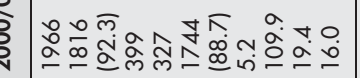

8

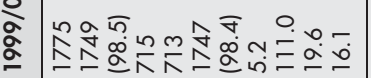

2

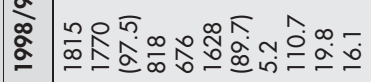

$\stackrel{\infty}{\circ}$

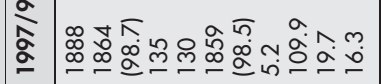

응

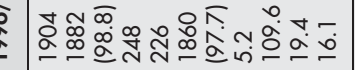

일

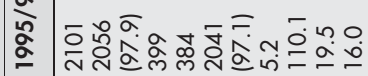

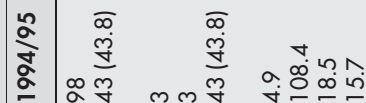

I

赵

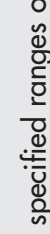

.

政

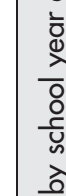

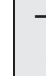

政

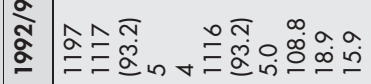

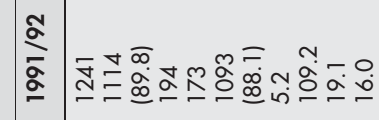

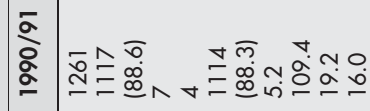

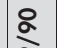

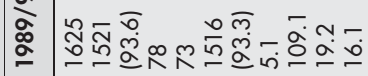

$\infty$

œ

$\infty$

离

这

总

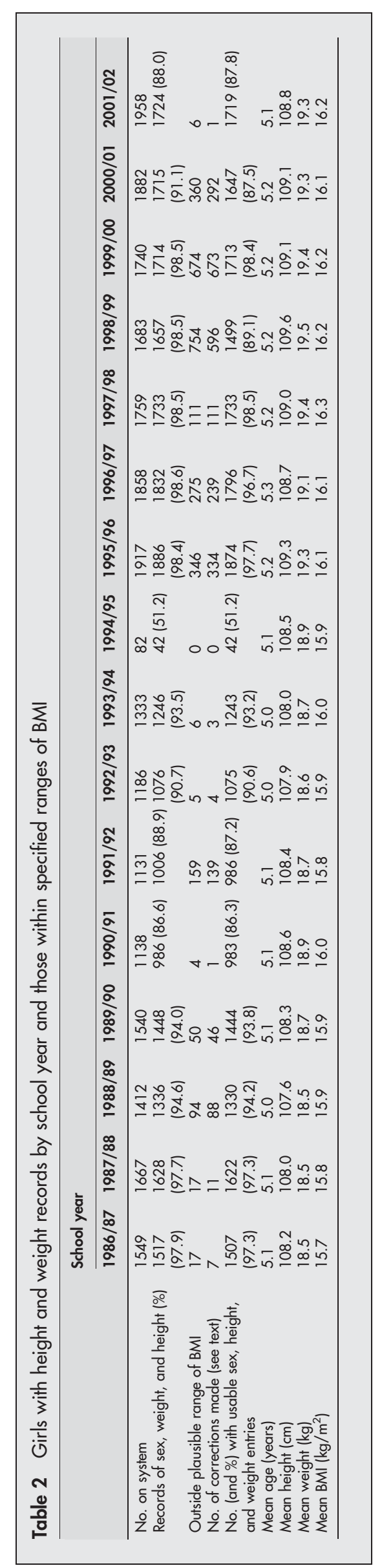

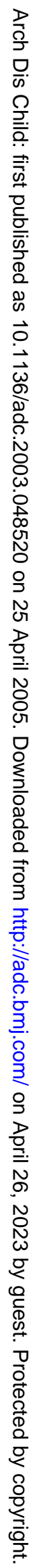




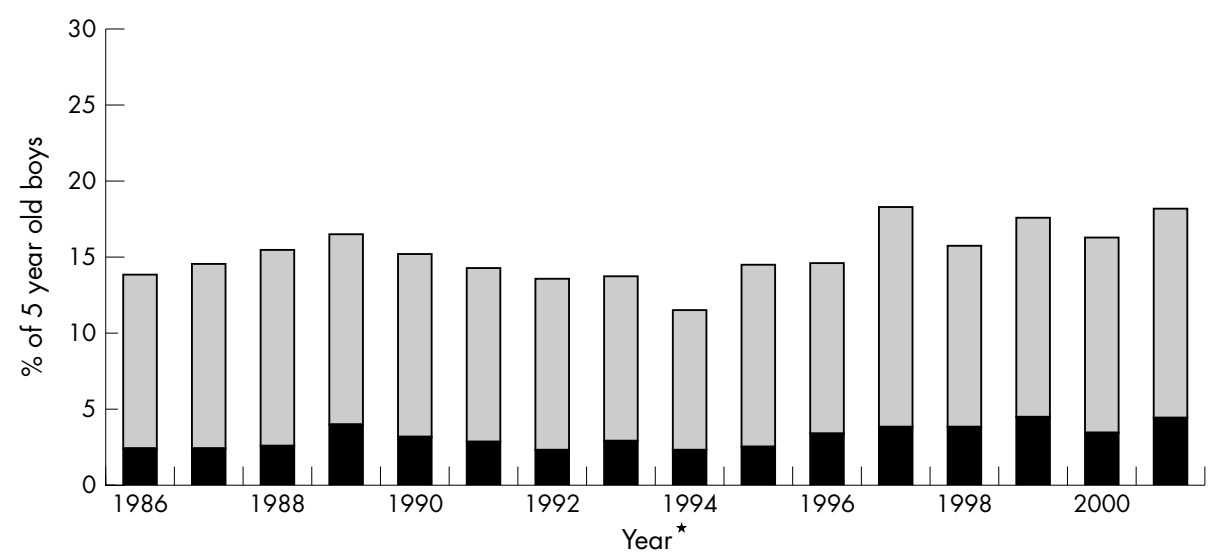

Figure 1 Percentages of boys (aged 5) by IOTF cut off points for being in overweight (grey) and obese (black) categories. *1994/95 based on 43 individuals only.

Data on the proportion of children in the various BMI categories (acceptable, overweight (but not obese), and obese) were analysed with logistic regression models using the $\mathrm{R}$ statistical environment. ${ }^{6}$ Time trends were initially investigated using "year" as a covariate, alongside a factor coding for additive differences between males and females. An interaction term was then included to test for any differences in the time trend parameters between males and females.

\section{RESULTS}

Tables 1 and 2 show, for boys and girls respectively, the number of children present on the Child Health Computer System (for example, for boys in 1986/87, $n=1640$ ), the number with any record of sex, height, and weight $(n=1611)$, the number with a calculated BMI outside the plausible range of $10-27(n=17)$, the number of records which could be brought within this plausible range by multiplying the recorded weight by $10(n=11)$, and the total number of BMI values thus deemed usable $(n=1605)$. Tables 1 and 2 also show the mean age, height, weight, and BMI values for each successive year.

With the exception of 1994/95 which, as noted below, was a year of transition between one system and another, overall coverage ranged between $86.8 \%$ (in 1997/98) and $98.5 \%$ (2001/02). The minimum and maximum coverage for boys were $85.7 \%$ (2001/2002) and 98.5\% (1997/98), and for girls, $87.2 \%$ (1991/92) and 98.5\% (1997/98). Mean height, weight, and BMI at the beginning of this period for boys were $109.2 \mathrm{~cm}, 18.9 \mathrm{~kg}$, and $15.8 \mathrm{~kg} / \mathrm{m}^{2}$ respectively, compared with $110.0 \mathrm{~cm}, 19.7 \mathrm{~kg}$, and $16.2 \mathrm{~kg} / \mathrm{m}^{2}$ at the end; for girls, the equivalent means were $108.2 \mathrm{~cm}, 18.5 \mathrm{~kg}, 15.7 \mathrm{~kg} / \mathrm{m}^{2}$, and $108.8 \mathrm{~cm}, 19.3 \mathrm{~kg}$, and $16.2 \mathrm{~kg} / \mathrm{m}^{2}$.

Figures 1 and 2 show the proportions of boys and girls respectively who were in the overweight (but not obese) and obese ranges of BMI according to the IOTF BMI cut off points for boys and girls of this age. The proportion of boys within "acceptable", overweight, and obese ranges, in 1986/87 were $86.2 \%, 11.3 \%$, and $2.5 \%$ respectively, while, in $2001 / 02$, these proportions were $81.7 \%, 13.7 \%$, and $4.6 \%$. For girls, the equivalent proportions were $82.9 \%, 13.5 \%$, and $3.6 \%$ in $1986 /$ 87 , and $73.5 \%, 19.5 \%$ and $6.9 \%$ in $2001 / 02$.

Logistic regression analysis showed that the proportion of overweight or obese children increased significantly over the study period $(\mathrm{p}<0.001)$. The proportion of overweight or obese girls was, as expected, significantly higher than that of boys, but the data also revealed that the rate of increase in this proportion was significantly higher for girls than boys $(p<0.01)$. The odds of being in either the overweight or obese categories increased at an annual rate of $1.6 \%$ for boys, compared to $2.9 \%$ for girls. The fitted model estimates a total increase in the proportion of overweight or obese individuals from $14 \%$ to $17 \%$ for boys and $19 \%$ to $26 \%$ for girls, over the 16 years of the study (illustrated in fig 3). Logistic regression of the proportion of children without valid sex, height, and weight measurements showed no relation with the year of study.

When the data are broken down into separate overweight and obese categories, the same time trends and sex differences can be seen. There is however, a further suggestion

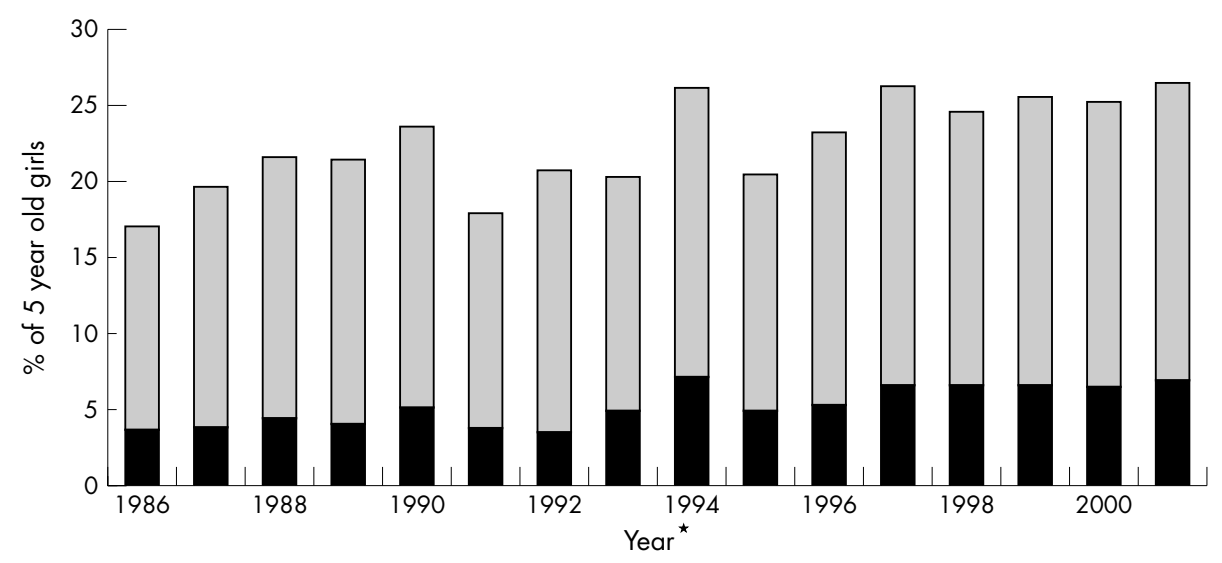

Figure 2 Percentages of girls (aged 5) by IOTF cut off points for being in overweight (grey) and obese (black) categories. *1994/95 based on 43 individuals only. 


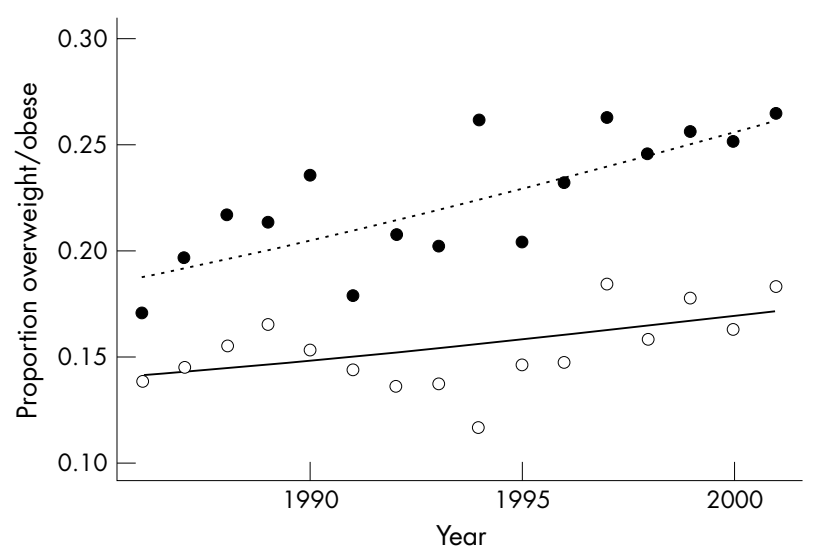

Figure 3 Fit of logistic regression model to time trends in proportions of overweight/obese boys (solid line) and girls (dotted line). The rate of increase is significantly higher for girls $(p<0.01)$.

that the largest effects are seen in the obese rather than overweight category. The estimated annual increases in odds of being in the overweight (not obese) range were $0.9 \%$ in boys (an increase that is not statistically significant) and $1.9 \%$ in girls. The annual increases in odds of being in the obese category were $3.4 \%$ and $4.8 \%$ for boys and girls respectively.

\section{DISCUSSION}

Coverage, in relation to height and weight data in these localities is far better than that reported elsewhere. Banerjee and colleagues, ${ }^{2}$ working in neighbouring areas, reported far lower coverage. They were also very critical of the system as a means of identifying individual children with growth problems. The use of the system explored here, the monitoring of a major public health problem on a population basis, is a very different issue. It is a use explicitly argued for in the commentary ${ }^{3}$ that accompanies that article.

With any population survey that does not have 100\% coverage, the issue of non-response bias must be considered. Given that, overall, the lack of coverage in this study was low $(6 \%)$, the magnitude of any non-response bias is likely to be small but might occur, for example, if children with particularly high (or low) BMI values were excluded for any reason. The reasons for exclusion from analysis included children not measured (either absent from school or when parental consent was not obtained) or those who were measured but whose data had been entered incorrectly and were not amenable to "correction" as described above. If there were an association between exclusion from analysis and particularly high or low BMI then one might expect a relation between the proportion excluded from analysis and time. No such relation was observed. The number of "corrections" that were made during the analysis also showed no discernible relation with time, and is, in our opinion, highly unlikely to have contributed to the trends observed. The exact ages at which the children were measured also shows no trend with time so that the rise in overweight and obesity with time cannot be attributed to the children being slightly older towards the end of the time period than at the beginning.

The only way in which, retrospectively, measurement errors can be identified is through the search for "shrinking children"; children who have been measured twice and whose second height measurement is less than the first. This occurred in approximately $20 \%$ of those double measurements. In cases such as these either the first or the second height measurement, or both, must be incorrect. Though a less than adequate means of identifying measurement errors, there was no obvious pattern of these particular errors over time.

The finding that overweight and obese children have increased in frequency in this population over this time scale is not unexpected. Five year old boys and girls in 2001/02 were, on average, $0.8 \mathrm{~kg}$ heavier than their predecessors in 1986/87, with boys having gained, on average, $0.4 \mathrm{~kg} / \mathrm{m}^{2}$ in BMI and girls $0.5 \mathrm{~kg} / \mathrm{m}^{2}$ over this time, with small $(0.8 \mathrm{~cm}$ and $0.6 \mathrm{~cm}$ respectively) gain in height. This modest average weight gain translates into an approximate doubling in the proportion classified as obese. The finding, from logistic regression analysis, that the trend in girls is significantly greater than that in boys may be important in relation to intervention.

Childhood obesity is a serious condition with serious consequences. Any method of monitoring trends in the population needs to be simple and accurate and to have comprehensive coverage. BMI is a proxy measure of obesity and, for various reasons, is not perfect ${ }^{5}$ but it is an acceptable and suitable measure for epidemiological purposes. Several studies have shown differences in BMI distribution between child populations even within the $\mathrm{UK}^{7}$ and suggest that obesity is more prevalent in more deprived populations. Wales has more social deprivation than most other European Union countries, and within the local authorities in this study there are several of the most deprived wards in Wales. No examination of the relation between overweight and obesity and deprivation can be made with the current system.

Examining trends and health inequalities such as this on a routine basis is a vital part of public health surveillance; given the resources already committed to measuring height and weight at school entry, to ignore this source would be a massive waste of resources. However, in order to maximise its use, full coverage must be achieved, as far as possible, and the quality of measurement and data entry need to be enhanced and maintained with a standardised quality enhancement programme. The availability of the data also needs to be improved, and to make it truly national in coverage, needs to be collated at a higher level than individual Trusts. The challenge now is to develop successful preventive measures directed at obesity in preschool and school children using sources such as this to track our success or otherwise in the future.

\section{Authors' affiliations}

S E Jones, M James-Ellison, Department of Child Health, Swansea NHS Trust, Swansea, UK

S Young, Information and Statistics Department, Swansea NHS Trust, Swansea, UK

M B Gravenor, R Williams, Professorial Unit of Clinical Epidemiology and Public Health, Swansea Clinical School, University of Wales

Swansea, Swansea, UK

Competing interests: none declared

\section{REFERENCES}

1 International Obesity Task Force. Childhood obesity: preventing and managing excess body weight during childhood. Report to the World Health Organisation. London: International Obesity Task Force, 2003.

2 Banerjee S, Morgan RJH, Rees SA, et al. Height screening at school: ineffective without high standards and adequate resources. Arch Dis Child 2003;88:477-80

3 Betts P. Commentary. Arch Dis Child 2003;88:480-1.

4 Hall DMB. Health for all children: report of the Third Joint Working Party on Child Health Surveillance. Oxford: Oxford University Press, 1996.

5 Cole TJ, Bellizzi MC, Flegal KM, et al. Establishing a standard definition for child overweight and obesity worldwide: international survey. BMJ 2000;320:1240-3.

6 Ihaka R, Gentleman R. A language for data analysis and graphics. J Comp Graph Stat 1996;5:299-314

7 Armstrong J, Dorosty AR, Reilly JJ, et al. Coexistence of social inequalities in undernutrition and obesity in preschool children; population based crosssectional study. Arch Dis Child 2003;88:671-5. 